FORMATION Formation emploi

Revue française de sciences sociales

122 | Avril-Juin 2013

Relever les défis de la Validation des Acquis de l'Expérience (VAE)

\title{
Postface : La VAE entre repli sécuritaire et développement des compétences
}

\author{
Yves Clot et Bernard Prot
}

\section{(2) OpenEdition \\ Journals}

Édition électronique

URL : http://journals.openedition.org/formationemploi/4010

DOI : 10.4000/formationemploi.4010

ISSN : 2107-0946

Éditeur

La Documentation française

Édition imprimée

Date de publication : 30 juin 2013

Pagination : 139-150

ISSN : 0759-6340

Référence électronique

Yves Clot et Bernard Prot, «Postface : La VAE entre repli sécuritaire et développement des compétences », Formation emploi [En ligne], 122 | Avril-Juin 2013, mis en ligne le 10 juillet 2013, consulté le 30 octobre 2020. URL : http://journals.openedition.org/formationemploi/4010 ; DOI https://doi.org/10.4000/formationemploi.4010 


\title{
Postface La VAE, entre repli sécuritaire et développement des compétences
}

\author{
Yves Clot \\ Psychologie du travail \\ Centre de Recherche sur le Travail et le Développement, CNAM (Conservatoire national des arts \\ et métiers) \\ BernaRd PROT \\ Psychologie du travail \\ Centre de Recherche sur le Travail et le Développement, CNAM (Conservatoire national des arts \\ et métiers)
}

Peut-on valider les acquis de l'expérience dans les termes d'une certification, qui doit également attester des acquis d'une formation? Si c'est le cas, dans quelles conditions est-ce possible ? À partir de la lecture des articles qui composent ce numéro, nous revenons sur deux résultats d'une série d'études que nous avons réalisées depuis 1998 à propos de la validation des acquis. Ainsi, nous interrogeons d'abord la nature des référentiels et des acquis à valider, puis le rôle du collectif de travail. Enfin, nous ouvrons la discussion sur le statut incertain du travail contemporain dans les instances chargées, en principe, de " sécuriser les parcours".

\section{Quels référentiels pour valider quels acquis?}

Considérons d'abord ce qui doit en principe être fait dans le dispositif de validation des acquis. La loi du 20 juillet 1992 reconnaît que "l'activité professionnelle est productrice de connaissances et de compétences». Cette idée, reprise lors de la généralisation du dispositif en 2002, stipule que la validation porte sur des " productions " professionnelles particulières.

L'expérience ne garantit pas le développement d'acquis. Les acquis peuvent se développer si le sujet reconsidère l'expérience vécue, les objectifs, les techniques employées, les réussites et les échecs, en s'appuyant sur d'autres points de vue, comme celui des collègues affectés à la même tâche, ou bien ceux du chef d'équipe, de l'ingénieur, du gestionnaire... Les conceptions quotidiennes se développent dans des "genres professionnels " reliés à des fonctions spécifiques et à l'histoire des collectifs et de l'entreprise. ${ }^{1}$

1. On entend par là, en référence à d'autres travaux en psychologie du travail (Clot, Faïta, 2001), une histoire 
Dès lors, il peut exister bien des types de validation. Dans un milieu de travail, on distinguera pour le moins la validation par les pairs, qui apprécieront la validité d'une technique originale dans le cadre collectif, de celle d'un des membres de la ligne hiérarchique, sur la base des critères qui relèvent de sa compétence. Qualité, quantité, sécurité, pénibilité... la production est réalisée, de plus en plus peut-être, au carrefour d'impératifs et de normes. Chacun peut développer une conception spécifique à sa fonction du bon compromis pour définir les critères du travail "bien fait ». On comprend donc bien les difficultés rencontrées par les spécialistes de la conception de référentiels de compétences en entreprise lorsqu'ils tentent de produire un document unique au milieu de conceptions et d'intérêts qui peuvent être divergents (Jouvenot, Parlier, 2005).

Mais la validation instituée par la loi en 2002 est encore d'un autre ordre. Son référentiel est externe à un milieu de production spécifique; il s'agit d'un référentiel national constitué, en principe, après "avis d'instances consultatives auxquelles participent les organisations représentatives d'employeurs et de salariés". C'est en tout cas une condition fixée par la loi pour l'inscription de la certification au Répertoire national des certifications professionnelles (RNCP). On est donc loin d'un référentiel de compétences d'entreprise ou encore du modèle anglais de référentiel de qualification défini "par les employeurs pour les employeurs", qui soutient la validation de skills, des "compétences fonctionnelles", selon les termes de Moncel (2012).

Il aurait pu en être autrement. Dans la période qui précède la loi de janvier 2002, Merle (2007) rappelle que les ministères, les organisations syndicales et patronales ont étudié différentes options, pour valider ces acquis, comme celle qui reposerait sur deux systèmes de validation distincts, l'un en fin de formation et l'autre pour l'expérience au travail. Mais à travers les "doutes " "hostilités " et " ambivalences" des différents protagonistes (pp. 58-59), la décision fut prise. On a conservé un seul système. Les diplômes associés aux certifications professionnelles des organismes de formation et de branches, inscrits dans un RNCP, constitueront la plateforme commune à la validation des acquis de l'expérience aussi bien qu'à la validation des acquis de la formation.

Dans le système français, l'accès à la certification par validation des acquis de la formation et l'accès à la certification par validation des acquis de l'expérience sont séparés car ils reposent sur deux référentiels distincts, mais ils ne peuvent pas être radicalement dissociés. En effet, le référentiel utilisé en validation des acquis de l'expérience doit permettre de déterminer vers quelles unités de formation se tourner en cas de validation partielle; il doit être cohérent avec l'évaluation de fin de formation pour définir ce qu'on attend d'un titulaire de ce diplôme, par exemple en termes de niveau de compétence par rapport à un titulaire d'un diplôme de niveau inférieur ou supérieur.

collective qui contribue à produire et renouveler des ressources techniques et langagières. 
Le travail de validation des acquis consiste donc à établir des correspondances entre des connaissances de nature très différentes. Ce dispositif peut alors favoriser une vaste confusion recouverte du voile opaque de la notion de compétence, si présente en tous lieux mais si peu définie.

La validation des acquis sera possible si on ne confond pas les acquis de la formation et les acquis de l'expérience. C'est le point de départ que nous avons adopté lors de nos études réalisées dès 1998 avec les services et des professionnels du ministère de l'Éducation nationale (Clot, Ballouard, Magnier, Werthe, 2000 ; Clot, Prot, Werthe, 2002). Son origine est dans la différence faite par Vygotski (1997) entre les " concepts quotidiens " qui se forment dans le "heurt avec les choses» et sont largement "non conscients", distingués des "concepts scientifiques " dont les définitions sont explicites et qui se développent volontairement "dans le processus d'enseignement». Les premiers doivent d'abord être fonctionnels quand les seconds permettent d'abord de distinguer des catégories, produire des analyses, établir des explications (p. 281).

Bien sûr, les concepts d'un référentiel d'emploi ne sont pas des " concepts scientifiques " et les " concepts quotidiens " d'un professionnel ne sont pas assimilables aux concepts quotidiens des enfants scolarisés qui étaient au centre de la réflexion des fondateurs d'une approche de l'apprentissage scolaire. Mais le modèle de pensée reste pertinent pour caractériser le travail en validation des acquis. Il permet de penser que le candidat à la VAE doit établir une correspondance entre les concepts quotidiens qu'il a développés au travail et les conceptions qui sont retenues comme des références partagées pour l'obtention du diplôme sur le plan national. Ce qui fait la force de ces concepts quotidiens, par exemple leur validité empirique, fait la faiblesse des concepts abstraits du référentiel et inversement, la force de ceux-ci, en particulier leur validité dans des contextes de travail différents, constitue la faiblesse des concepts quotidiens.

Si on les distingue, alors on peut montrer, comme nous l'avons fait, que le candidat qui les met en correspondance, à travers l'élaboration du dossier, dans les entretiens avec des accompagnateurs ou avec des jurys, peut " réaliser ", au sens psychologique du mot, qu’il a développé, ou qu'il n'a pas suffisamment développé, certaines connaissances, certaines règles, certains principes organisateurs de l'action professionnelle, certaines techniques de travail. Le candidat forme alors une pensée hybride ; il s'engage dans une activité d'abstraction, à la fois fondée sur son expérience et sur une définition d'une technique, d'une tâche, d'une procédure, d'une règle de métier... qui relève d'un autre niveau de généralité.

On dira qu'il forme un "concept potentiel» (Vygotski, 1997 ; Prot, 2003 ; 2012). Le candidat peut envisager autrement son expérience, à partir de cette signification sociale qui, dans un premier temps, pouvait lui être étrangère. Il peut ainsi saisir ce qu'il « savait sans le savoir ", ou bien comprendre que ses acquis en la matière ne sont pas suffisants et qu'il pourrait trouver un intérêt à approfondir sa connaissance du concept de référence, des connaissances «théoriques " qui le soutiennent. La validation des acquis devient possible si ce travail d'élaboration permet au candidat de faire apparaître dans son dossier et durant 
l'entretien éventuel des expériences "significatives ", assorties des analyses qu'il en fait, à partir desquelles le jury peut prendre la mesure du niveau de développement des concepts quotidiens du candidat.

La validation n'est donc pas une simple reconnaissance sociale adressée au travailleur. Elle requiert aussi une transformation de l'expérience qui est pensée dans ce nouveau contexte d'action. L'acquis est un développement de l'expérience. Les modèles interactionnistes d'étude de la VAE pourraient avoir quelques difficultés à expliquer ces transformations. La validation n'est pas seulement " mise en scène de la vie quotidienne dans un jury ", parce que la scène fait dialoguer des références discordantes et que la discordance est créatrice (Clot, Prot, 2003).

C'est à travers la discordance des points de vue que les acquis montrent s'ils conservent une validité au-delà du milieu d'exercice initial. On peut dire que les concepts quotidiens du travail sont transférés dans l'activité de validation, ils changent de fonction puisqu'ils deviennent un moyen argumentatif adressé au jury, en même temps qu'ils entrent dans une "zone de développement potentielle " pour le candidat confronté à les penser de manière plus "générale ". Cette démarche suscite également un développement inverse chez les accompagnateurs et les membres du jury, lorsqu'ils cherchent à établir la correspondance entre leurs points de vue et les événements rapportés par le candidat.

Pour le candidat, cette démarche n'est pas spontanée. Dans ce numéro, le texte de P. Saielli et M.-O. Legrand souligne la différence des « logiques », comme par exemple une recherche de reconnaissance personnelle, dans lesquelles les candidats peuvent "sinvestir " au point de risquer de "fermer» ce qui constitue un «espace transitionnel». Il serait sans doute possible de discuter plus précisément pour définir quel est « l'objet » qui se constitue dans cet espace social original, et sur lequel porte justement l'évaluation, afin de mieux distinguer la validation des acquis et la formation professionnelle. Mais on voit bien dans ce texte que le travail des accompagnateurs et des jurys est déterminant dans cette élaboration référencée de l'expérience vécue au travail, surtout pour des travailleurs dont les milieux quotidiens ne favorisent pas une telle élaboration. C'est là une autre condition pour valider les acquis.

\section{2uel collectif de travail en validation des acquis?}

L'expérience de Validation des acquis professionnels accumulée au ministère de l'Éducation nationale avait ouvert la voie en matière de méthode. D. Ravat (1997) tirait le bilan de cette expérience en mettant en évidence les deux piliers de la validation des acquis à la française : sur le plan politique, promouvoir l'égalité d'accès au diplôme par un dossier, de sorte que le candidat puisse accéder à la validation, quelle que soit sa situation au regard de l'emploi et de son éventuel employeur, et en prenant en compte l'ensemble de ses expériences professionnelles; sur le plan de la méthode, accompagner le candidat dans 
son travail d'analyse et d'élaboration de l'expérience, parce que les acquis sont largement non conscients, au point que le candidat peut les sous-estimer ou les surestimer, d'autant qu'il lui faut se familiariser avec les exigences spécifiques d'un diplôme. Principe et méthode d'analyse sont donc indissociablement liés pour ouvrir une nouvelle voie d'accès aux diplômes professionnels en passant par l'analyse des expériences.

Pour mettre en œuvre une telle méthode de validation des acquis, les conseillers, les accompagnateurs, les jurys doivent produire, eux aussi, un "genre professionnel» spécifique à la situation de VAE (Clot, Prot, Werthe, 2000).

Mais en VAE, l'existence d'un genre professionnel relève d'un esprit collectif assez particulier, disons qu'il doit relier des points de vue hétérogènes. Il doit être spécifique à chaque étape du dispositif, afin de ne pas mélanger information initiale, accompagnement du candidat et jury (Werthe, 2003). Cepentant, la séparation des fonctions ne doit pas empêcher l'existence d'un collectif partagé entre eux tous, par lequel le candidat comprendra qu'il n'est pas dans une situation d'enseignement, de formation continue, de jury d'examen, qu'il n'est pas en bilan de compétences ou en entretien de conseil de carrière ou d'orientation. Tenter d'établir des correspondances entre l'expérience et la certification constitue le coeur du métier spécifique et commun à tous ; il suppose de « fabriquer » des techniques qui permettent d'analyser l'expérience au travail pour y trouver les éléments significatifs au regard du diplôme visé. Des accompagnateurs eux-mêmes ont montré comment leur technique repose sur la coopération entre un "accompagnateur spécialiste " du domaine d'emploi et un "accompagnateur généraliste ", attentif aux obstacles inhérents à l'élaboration et la formalisation de l'expérience dans le dossier (Lucas, Retières, 2007).

Mais, comme le révèlent plusieurs articles de ce numéro, les conditions d'emploi des conseillers comme celles des accompagnateurs sont parfois peu favorables à la stabilité des personnels, à la constitution patiente et forcément longue d'une histoire technique partagée, particulièrement lorsqu'elles relèvent d'un marché régulé par des logiques d'appel d'offre et de sous-traitance.

L'investissement des " grands " organismes certificateurs permet-il de compenser en partie cette déperdition des énergies ? Le texte de P. Mayen et de J.-P. Pin, dans ce numéro, montre une coopération de plusieurs années entre les professionnels du ministère de l'Agriculture et les chercheurs d'Agrosup, en lien avec un fond de formation régional, qui témoigne d'avancées dans cette direction. Il souligne l'importance du montage institutionnel qui permet à la validation des acquis d'exister en tant que "situation " de VAE permettant au candidat de "travailler sur ce qu'on fait tout les jours ». La constance d'une politique institutionnelle et interinstitutionnelle semble donc non seulement souhaitable mais aussi possible. Une récente publication en témoigne, avec l'exemple d'une institution de formation suisse (Cortessis, Salini, Rywalski, 2013). Les contributions de R. Belisle (2011) apportent également des analyses sur ce point, depuis le Québec. 
L'investissement institutionnel peut aussi faire défaut. Ce fut le cas dans notre expérience avec le ministère chargé de la Jeunesse et des Sports. Une longue période de structuration d'un collectif national composé des experts en VAE de chaque région, sous l'égide du ministère, a permis de favoriser des liens " remontants " autant que les liens " descendants " entre les sites locaux et l'administration centrale. Plusieurs textes rendent compte de la coopération entre chercheurs et des professionnels qui ont commencé à faire connaitre leurs propositions autour des conditions concrètes de réussite d'un processus de VAE, depuis l'information au jury en passant par l'accompagnement et la formation des différents acteurs (Alory, 2007 ; Besson, 2007 ; Lainé, 2007). Pourtant, la restructuration des services et la réorientation des politiques de ce ministère, à la fin des années 2000, ont mis à mal les conditions de l'élan collectif.

L'ouverture systématique des diplômes de l'enseignement supérieur soulève, sur ce point, de vastes interrogations... Comment envisager que les institutions universitaires auraient la capacité de soutenir la construction des processus collectifs de travail en VAE sur un ensemble potentiel de 12000 titres, d'autant plus dans les circonstances actuelles ? La construction de référentiels à elle seule constitue une difficulté majeure pour une formation universitaire ; il en va de même de l'évaluation des rapports entre le diplôme et les "besoins sur le marché du travail ", ou encore de la définition des critères et des modalités de mesure d'une " réalité de l'insertion » en sortie de formation, qui sont requises par la Commission nationale des certifications professionnelles (CNCP).

Les études du Céreq ont largement contribué à révéler que la relation entre formation et emploi n'est pas une relation de type "adéquationniste " et que l'étude de cette relation soulève une difficulté singulière dans l'enseignement supérieur, caractérisé par l'affluence de titres. Une série de travaux sociologiques, économiques, juridiques portant sur les transformations induites par la généralisation de la VAE, et plus largement par l' "injonction à la professionnalisation des diplômes", soulignent que l'action formative et certificative est traversée par de nombreux paradoxes (Maillard, 2012).

Le "rapprochement $"^{2}$ tant attendu entre travail et diplôme est pourtant possible. De notre point de vue, il devient possible lorsqu'on prend acte de la différence entre les acquis de la formation et les acquis du travail et qu'on cherche à construire une correspondance entre eux qui repose sur le principe de leur développement réciproque. Ce modèle de correspondance est alors un modèle dialogique, au sens fort du mot. L'important travail réalisé sur la construction des diplômes professionnels par G. Brucy (1998) en apporte de belles démonstrations historiques. Avec sa perspective sur la longue

2. On trouve une telle expression dans les propos de Georges Asseraf, président de la CNCP, interviewé par Saïma Kadri, à l'occasion des dix ans de la loi de 2002 : «Le centre de gravité du diplôme sest déplacé du savoir aux compétences en lien avec l'activité professionnelle». Document en ligne sur le site de la CNCP : http://www. cncp.gouv.fr/gcp/pages/site/cncp-internet-cncp/lang/fr/Accueil35701 
durée et à travers de très nombreux exemples, l'historien montre comment la controverse peut être centrale dans la création et la rénovation des diplômes; et ce, non seulement entre l'État, les syndicats et les employeurs, mais souvent au sein même de chaque camp, à propos du travail attendu des titulaires de diplômes comme au sujet de la formation souhaitée. Les désaccords deviennent alors le foyer potentiel d'une rénovation régulière du diplôme et des formations. Le travail de conception d'un diplôme participe ainsi à l'histoire de la branche professionnelle.

Mais les textes du présent numéro s'inscrivent aussi dans une telle correspondance, avec des approches centrées sur le fonctionnement de la VAE. Ainsi, S. Bellini propose de considérer qu'un jury doit constituer une "norme d'évaluation " lorsqu'il est confronté à ces "savoirs" d'expérience si différents des savoirs enseignés. P. Saielli et M.-O Legrand, s’appuyant sur une enquête participante, considèrent que la VAE est le lieu d'un "partage " entre "culture académique " et "culture professionnelle ", à travers des "débats " qui s'avèrent "constitutifs d'une définition de la situation de jury".

Le référentiel n'est pas une "grille " qu'il suffirait d'appliquer sur le dossier du candidat, dans une illusion adéquationniste. On peut considérer le référentiel dans une approche dialogique : il est un instrument d'analyse pour ce candidat lui-même, un moyen d'établir la liaison entre différentes manières de " connaître " le travail, celles qui structurent les situations professionnelles et aussi les connaissances théoriques enseignées en formation. Cette approche dialogique de la validation des acquis permet aussi de penser que l'activité du jury consiste à produire une décision juste lorsque les divergences de points de vue des évaluateurs leur permettent de s'affranchir de leurs préjugés sur le candidat et sur le travail.

La conception et la rénovation des référentiels deviennent alors déterminantes. Elle sont souvent engagées dans une "démarche compétence ", démarche dont il a pourtant été suffisamment rappelé qu'elle reste trop floue pour fonder des processus de qualification (Zarifian, 2001). Dans une perspective économique, A. Vinokur (2002) a considéré le déploiement massif de référentiels comme la manifestation et le moyen d'un «changement sans réforme".

En matière de déploiement massif de la VAE et de réforme, la contribution de M. Santos, qui rapporte l'expérience peu étudiée jusque-là du Portugal, est tout à fait édifiante. Lapproche historique des institutions éducatives y est abordée d'emblée pour souligner qu'après la période de la dictature, «le domaine des politiques éducatives concernant les adultes a été systématiquement ignoré", jusqu'à la mise en place d'une politique gouvernementale très volontariste pour instaurer la VAE, assortie de moyens financiers conséquents. Et c'est justement l'analyse de l'activité de formateurs impliqués dans ces actions qui révèle comment naissent, à ce niveau, les fortes contradictions entre le volontarisme national, la prescription à la "décentralisation" des dispositifs, en même temps que "la singularisation et la contextualisation des parcours de formation ". 


\section{La VAE entre sécurité individuelle et travail}

En France, en vingt ans, la validation des acquis a progressé dans les institutions. Du CAP au BTS (respectivement certificat d'aptitude professionnelle et brevet de technicien supérieur), elle a été déployée vers l'enseignement supérieur ; tous les ministères certificateurs ont désormais construit leur système de validation. Le rapport 2012 de la CNCP fait état de 2872 certifications enregistrées sur la demande d'organismes de formation ou de branches professionnelles, ce qui porte à plus de 7700 le total des certifications accessibles à la VAE. Une sorte d'autoroute institutionnelle traverse désormais ce qui était parfois considéré comme le " maquis des acquis» (Liétard, 1997).

Mais les études statistiques montrent une tout autre géographie de la pénétration de la VAE dans les milieux professionnels. Elle n'augmente pas, elle est concentrée sur certains secteurs. Le nombre de candidats présentés ne dépasse guère 50000 depuis 2007, malgré une mise en place désormais généralisée à tous les certificateurs. En 2011, dix diplômes réunissent à eux seuls la moitié des titres demandés et parmi eux neuf relèvent du secteur sanitaire et social, la proportion de femmes peu diplômées y étant très importante.

Si la VAE répond à une mission d'élévation de niveau de diplôme dans ce secteur et quelques autres comme l'emploi administratif, elle ne soutient guère les transformations industrielles et des services, qui sont pourtant au cœur des enjeux actuels de "sécurisation des parcours ». N'assiste-t-on pas à un divorce entre le dispositif et ses enjeux affichés ?

La rupture est peut-être déjà engagée depuis 2002. Un enthousiasme, souvent un militantisme, aux racines sociales et politiques très hétérogènes, a soutenu l'adoption de la loi, comme le rappelle un article de Lochard (2007) qui montre la congruence de mouvements sociaux qui entendaient obtenir la "dignification» de ces acquis souvent maltraités dans notre société. Mais ce but est atteint alors que les pouvoirs publics ont engagé une politique "pragmatique ", pour tenter de "régler des questions de qualification et d'emploi " (p. 92), dont la VAE sera un élément central. Si la VAE est "triomphante " au regard du très vaste consensus exceptionnel en France, Neyrat (2007) soutient l'idée que le consensus repose sur le modèle individualiste et économique de la formation, de l'orientation et, désormais, de la certification. Sans doute les professions les plus " assises " vontelles s'organiser pour limiter le recours à une " marchandisation " de l'accompagnement et de la certification qui est radicalement opposée aux principes "d'éducation permanente " affirmés par Condorcet, auxquels se référaient pourtant les mouvements qui ont soutenu l'émergence de la loi (p. 159).

Les textes de ce numéro contribuent à alimenter la réflexion sur ces importantes discordes relatives aux enjeux de la VAE. Celui de P. de Rozario notamment, puisqu'il souligne la "politique inédite en France par son ampleur et les moyens déployés" pour transformer l'accès aux diplômes dans le travail social. Ce texte montre particulière- 
ment l'importance des "facteurs d'organisation et de gestion de la VAE", parmi lesquels la dimension régionale prend une place conséquente. Il souligne ainsi l'intensité de l'engagement des participants, "les partenaires sociaux, les écoles de travail social et les certificateurs" dans les discussions visant à déployer "une telle politique de diplômation massive par la $V A E »$, tout en s'interrogeant sur les enjeux d'une telle mobilisation.

Si le travail social relève des professions les plus "assises ", pour reprendre le terme de Neyrat, la participation des branches professionnelles au déploiement de la VAE est souvent bien plus limitée, comme en atteste la faiblesse des effectifs de candidats mentionnée plus haut. Déjà lors des négociations qui ont précédé l'adoption de la loi, $\mathrm{V}$. Merle relève que les organisations patronales et syndicales n'ont "pas vraiment " montré " un grand intérêt pour leur implication dans le processus de régulation du système de certification" (2007, p. 58).

Limplication des employeurs et des salariés dans les négociations et dans la mise en œuvre de ces réformes soulève donc des interrogations.

C'est d'abord sous l'angle de la sécurité au regard de l'emploi que les enjeux sont définis ; on institue des "droits individuels " depuis la loi de 1991 sur le bilan de compétences, puis sur les congés de formation et désormais sur l'accès à la certification. Le statut de l'activité qui s’y réalise peut alors devenir incertain. Ainsi P. Mayen et J.-P. Pin, ici, écrivent que la VAE «s'apparente » à une "situation professionnelle ", même si "ce n'est pas une situation de travail». La VAE devient-elle alors une affaire individuelle ? Pourtant, comme bien d'autres dispositifs de la formation et de l'orientation à l'âge adulte, la VAE relève du droit du travail, les financements sont gérés par les organisations professionnelles dans des contextes en principe paritaires au niveau des régions, les salariés et les employeurs doivent en principe participer à l'élaboration des référentiels à l'aune desquels on évalue les acquis.

On peut considérer qu'un candidat à la VAE qui analyse son expérience, qui tente de " transférer » ses compétences vers d'autres fonctions, ou de les valider par un nouveau diplôme, continue de faire son métier. On peut même dire qu'il le développe. Si la dimension personnelle de l'implication en VAE est forte, c'est aussi parce qu'elle suppose un travail important sur l'expérience au travail. C'est donc un travail " au carré ». On peut développer " son métier " personnel, au sens psychologique (Clot, 2008) même lorsqu’on envisage de changer de fonction dans une entreprise ou même lorsqu'on veut changer de "métier" au sens sociologique.

Il est possible que la mise en oeuvre de la VAE participe à conforter un esprit sécuritaire tourné vers la protection de l'emploi et des " parcours » invididuels par l'accès à des certifications de niveau plus élevées. Pourtant, on ne parviendra pas à mettre les compétences en sécurité dans des diplômes ou dans des "portefeuilles de compétences ", quels qu'ils soient. Les compétences restent productives si elles sont employées. Les candidats sontils considérés comme des travailleurs qui prennent soin de leur expérience ou comme des individus qui gèrent leur parcours ? Ce sont les liens entre le travail et les différents 
emplois, entre les acquis des expériences vécues et les expériences à vivre, qui peuvent s’y développer ou s'y dissocier. Au-delà des formules univoques, utiles pour ouvrir la réflexion, le statut social de l'activité des candidats à la VAE mérite d'être discuté.

Les employeurs et les salariés qui s'engagent dans l'élaboration de référentiels sont concernés au premier chef. La participation des différents niveaux hiérarchiques et des employeurs est une épineuse question à laquelle les publications récentes qui portent sur l'élaboration de référentiels se mesurent, en gestion (par ex. Jouvenot et Parlier, 2005 ; Cavestro, Durieux, Monchatre, 2007), en formation (par ex. les textes du dossier du numéro 64 de Recherche et Formation, 2010) ou en certification (Maillard, 2012). Pour conserver un lien vivant entre les référentiels et l'activité au travail, on propose de faire porter la conception de ces référentiels sur les questions vives que soulève la réalisation d'un produit ou d'un service, sur les dilemmes qu’on y rencontre et sur les ressources développées par les collectifs pour tenter de faire et de renouveler leur travail (Prot, 2011 ; 2014). Sur ce point aussi, méthodes d'analyse et approches théoriques ont fort à faire.

Le repli sécuritaire sur l'emploi n'est pas la seule voie possible. La VAE appartient à une série de réformes qui nous incitent à étudier plus précisément les conditions qui permettent à chacun de développer son métier en dehors des lieux de production de biens et de services (Ouvrier-Bonnaz, Prot, 2007), dans ces espaces de «transition " professionnelle (Reille-Baudrin, 2012) au statut social souvent incertain. Il s'agit de chercher à quelles conditions les professionnels peuvent relier la réalisation d'un travail de qualité (Clot, 2012) avec leurs emplois, en instituant des références communes à propos du travail, en dehors de chaque emploi. Il s'agit de garder, au-delà de chaque personne, le travail vivant en chaque personne. Par l'inventaire qu'il propose, le présent numéro contribue à penser ces questions contemporaines. Que chacun des auteurs en soit ici remercié.

\section{Bibliographie}

Alory M. ( 2007), « Une pratique d'accompagnateur en VAE : le retour sur le parcours du candidat », Education Permanente, 171, pp. 105-116.

Bélisle R. (2011), "Québec: An overview of RAC/RPLC research since 2002", in Harris J., Breier M. and Wihak C. (dir.), Researching the recognition of prior learning. International perspectives, pp. 85-105, Londres: NIACE.

Besson M. (2007), « De l'analyse de l'activité en jury à la formation de jurys en validation des acquis : l'histoire d'une migration ", Education Permanente, 171, pp. 117-126.

Brucy G. (1998), Histoire des diplômes de l'enseignement technique (1880-1965), Paris, Belin.

Clot Y. (2008), Travail et pouvoir d'agir, Paris, PUF.

Clot Y. (2010), Le travail à coeur, Paris, La Découverte. 
Clot Y., Ballouard C., Magnier J., Werthe C. (2000), La validation des acquis professionnels : concepts, méthodes, terrain, Rapport d'étude à la Direction de l'enseignement scolaire, MEN, coll. "CPC documents».

Clot Y., Faïta D. (2000), « Genre et style en analyse du travail », Travailler, 4, pp. 7-42.

Clot Y., Prot B. (2003), " De l'analyse du travail à la validation des acquis ", L'Orientation scolaire et professionnelle, 2. (en ligne : http://osp.revues.org/index2711.html).

Clot Y., Prot B., Werthe C. (2002), La validation des acquis au milieu du gué, Rapport d'étude à la Direction de l'Enseignement scolaire, MEN, coll. « CPC Documents ».

Cortessis S., Salini D., Rywalski P. (2013), "Se former au conseil et à l'accompagnement en RVAE. Entre incertitude et projets ", Education Permanente, Hors série IFFP-IUFFP-EHB.

Jouvenot C., Parlier M. (2005) (s/d.), Elaborer des référentiels de compétences, Lyon, Editions de l'ANACT.

Laîné A. (2007), "Quand le sens de l'activité ordinaire change d'orientation ", Education Permanente, 171, pp. 127-136.

Liétard B. (1997), "Se reconnaître dans le maquis des acquis ", Education Permanente, 133, pp. 65-73.

Lochard Y. (2007), «L'avènement des savoirs expérientiels " Revue de l'Ires, 55, pp. 79-95.

Lucas J.-M., Retières J.-F. (2001), "Les activités singulières sont-elles solubles dans les référentiels ? ", Education Permanente, 146, pp. 151-160.

Maillard (2012) (s/d.), Former, certifier, insérer. Effets et paradoxes de l'injonction à la professionnalisation des diplômes, Rennes, PUR.

Moncel N. (2012), « Enjeux de l'articulation entre conception des diplômes et valorisation sur le marché du travail / l'exemple anglais ", in Maillard F., Former, certifier, insérer. Effets et paradoxes de l'injonction à la professionnalisation des diplômes, pp. 111129, Rennes, PUR.

Merle V. (2007), « Genèse de la loi de janver 2002 sur la validation des acquis de l'expérience ", Revue de l'Ires, 55, pp. 43-78.

Neyrat F. (2007) (s/d.), La validation des acquis de l'expérience. La reconnaissance d'un nouveau droit, Bellecombes en Bauges, Editions du croquant.

Ouvrier-Bonnaz R., Prot B. (2007) (Coord.), « Activité d'orientation et développement des métiers ", Education Permanente, 171.

Prot B. (2003), «Analyse du travail des jurys en validation des acquis : l'usage du référentiel ", L'Orientation scolaire et professionnelle, 32-2, pp. 219-243. (en ligne : http://osp. revues.org/index2711.html). 
Prot B. (2012), Former un concept potentiel pour valider les acquis de l'expérience, Thèse de doctorat. Sarrebruck : Editions Européennes.

Prot B. (2011), «Apprentissage de la conduite et sécurité routière : Un dilemme de référence pour la conception d'un référentiel de diplôme d'enseignant ", Activités, 8(2), pp. 189-201, http://www.activites.org/v8n2/v8n2.pdf.

Ravat D. (1997), "La validation des acquis. Pourquoi ?Comment ? ", Education Permanente, 13-4, Supplément Education nationale, pp. 5-11.

Reille-Baudrin E. (2012), "Conflits et mobilité dans l'espace d'une transition ", Education Permanente, Hors série AFPA, pp. 59-67.

Vinokur A. (2002), «Enseignement supérieur : un changement sans réforme », Formation Emploi, 79, pp. 19-30.

Vygotski L. (1997 1934), Pensée et langage, Paris, La Dispute.

Werthe C. (2003), "L'évaluation collective : l'exemple d'un jury de validation des acquis ", L'Orientation scolaire et professionnelle, 32-2, pp. 245-268. (en ligne : http://osp.revues. org/index2711.html )

Zarifian P. (2001), La logique compétence, Paris, Editions Liaisons. 